\title{
Modelling Fluid Flow and Heat Transfer in a Saturated Porous Medium
}

D.A. NIELD

d.nield@auckland.ac.nz Department of Engineering Science, University of Auckland, Private Bag 92019, Auckland, New Zealand

(Dedicated to A. McNabb on the occasion of his 70th Birthday)

\begin{abstract}
Since the days of Darcy, many refinements have been made to the equations used to model single-phase fluid flow and heat transfer in a saturated porous medium, to allow for such basic things as inertial effects, boundary friction and viscous dissipation, and also additional effects such as those due to rotation or a magnetic field. These developments are reviewed.
\end{abstract}

Keywords: porous medium, fluid flow, heat transfer

\section{Introduction}

Shenoy (1994) gives a two-page list of applications of the present subject under the headings Biomechanics, Ceramic engineering, Chemical engineering, Food technology, Geophysics, Groundwater hydrology, Industrial engineering, Mechanical engineering, Petroleum engineering, Soil mechanics.

A porous medium is a fixed (or almost fixed) solid matrix with a connected void space through which a fluid can flow. The fraction of void space to total volume is called the porosity. Most naturally occurring porous media have porosities less than 0.6 (an exception is hair), but man-made materials, such as metallic foam, can have porosities up to 0.99 .

The observations of Henry Darcy (1856) of the water supply at Dijon, and experiments on steady state unidirectional flow suggested Darcy's law, which in its refined modern form can be expressed as

$$
\partial p / \partial x=(\mu / K) v,
$$

where $\partial p / \partial x$ is the pressure gradient, $v$ is the filtration velocity, $\mu$ is the fluid viscosity and $K$ is the permeability (units length squared). The filtration velocity $v$ (velocity averaged over the medium) is related to the intrinsic velocity $V$ (velocity averaged over the pore space) by $v=\phi V$, where $\phi$ is the porosity. The permeability $K$ depends on the pore size (or particle diameter) $D_{p}$, the porosity, and also on the detailed geometry. A useful estimate is given the Carman-Kozeny relationship

$$
K=\frac{D_{p}^{2} \phi^{3}}{180(1-\phi)^{2}}
$$

Darcy's law means that the drag is proportional to the velocity. This holds for small velocities (Reynolds number, based on the pore scale, less than unity). It 
breaks down for larger velocities. Dupuit (1863), and Forchheimer (1901) found empirically that for larger velocities the drag is a quadratic function of the velocity. (I simplify the matter; a detailed historical account has been given by Lage (1998).)

\section{The Brinkman Forchheimer equation}

A modern refinement (see e.g. Hsu \& Cheng (1990), Vafai \& Kim (1990)) is the equation

$$
\rho\left[\frac{1}{\phi} \frac{\partial \mathbf{v}}{\partial t}+\frac{1}{\phi^{2}}(\mathbf{v} \cdot \nabla) \mathbf{v}\right]=-\nabla p+\mu_{e} \nabla^{2} \mathbf{v}-\frac{\mu}{K} \mathbf{v}-\frac{c_{F} \rho}{K^{\frac{1}{2}}} v \mathbf{v} .
$$

This applies to an incompressible fluid of density $\rho$. Here $v$ denotes $|\mathbf{v}|$, the magnitude of the Darcy velocity, while $\mu_{e}$ is an effective viscosity and $c_{F}$ is a dimensionless Forchheimer coefficient. The inertial terms (on the left-hand side) result from formal averaging. The first viscous term is the Brinkman term. The last term is the Forchheimer term. We now consider the significance of the various terms in Eq. (3).

1. The local time-derivative inertial term.

This is derived on the assumption that a spatial averaging process commutes with a derivative with respect to time. This breaks down when the porous medium has macroscopic structure such as a system of tubes. The decay of a transient is more rapid in narrow tubes than in wide tubes. Nield (1991) suggested that in this case $(1 / \phi) \partial \mathbf{v} / \partial t$ be replaced by $\mathbf{c}_{a} \cdot \partial \mathbf{v} / \partial t$, where $\mathbf{c}_{a}$ is a constant tensor (that is determined mainly by the nature of the pores of largest cross-sections). In any case, the ratio of the time-derivative term to the Darcy resistance is $\mathbf{c}_{a} \rho K / \mu T$, where $T$ is a characteristic time of the process being investigated, and this ratio is normally very small.

2. Advective inertial term.

Joseph, Nield and Papanicolaou (1982) argued that, when modelling dense media, the advective term involving $(\mathbf{v} \cdot \nabla) \mathbf{v}$ should be omitted because the inertial effects are already accounted for in the quadratic drag term involving $v \mathbf{v}$. This arises as a result of form drag on the solid particles. The drag is independent of the viscosity and acts in a direction opposite to $\mathbf{v}$. Nield (1991) argued that the inclusion of the $(\mathbf{v} \cdot \nabla) \mathbf{v}$ term leads to the prediction that longitudinal momentum can, unimpeded by the fixed solid matrix, be transmitted transversely, in conflict with expectation based on basic physics.

This is related to the difficulty of spin-up (by just rotating a solid boundary), and the absense of true macroscopic turbulence (involving a cascade of energy from large eddies to smaller eddies), in a dense porous medium. The averaging process leads to misleading results because it leads to a loss of vital information about the way in which the geometry of the solid matrix affects the flow by reducing the coherence of the fluid momentum pattern. 
One has a vector identity $(\mathbf{v} \cdot \nabla) \mathbf{v}=\nabla\left(v^{2} / 2\right)+\mathbf{v} \times(\nabla \times \mathbf{v})$. It was noted by Nield (1994) that at least the irrotational part, $\nabla\left(v^{2} / 2\right)$, of $(\mathbf{v} \cdot \nabla) \mathbf{v}$ needs to be retained in order to account for the phenomenon of choking in the high speed flow of a compressible fluid, but he suggested that the rotational part, involving the intrinsic vorticity, be deleted. His argument was based on the expectation that a medium of low porosity will allow scalar entities like fluid speed to be freely advected, but will inhibit the advection of vector quantities like vorticity. Nield and Bejan (1999) went a step further, and suggested that even when vorticity is being continuously produced (e.g. by buoyancy) one would expect that it would be destroyed by a momentum dispersion process due to the solid obstructions.

An argument providing further support for this point of view was presented by Nield (2000a). There are some subtleties about the effect of the inertial terms on motion in a porous medium. The power of the total drag force (per unit volume) is equal to the rate of viscous dissipation (per unit volume); for a detailed discussion see Nield (1999). The Forchheimer drag term, although it appears to be independent of the viscosity, contributes to the viscous dissipation. The effect of inertia is mediated via a change in the pressure distribution and the velocity distribution. The flip side of the coin is that when one closes the system of equations by introducing a Forchheimer drag term one should not assume that the convective inertia term that remains in the momentum equation is identical with that obtained by formal volume-averaging. After integration, it should lead to the correct expression for the averaged kinetic energy, which involves the magnitude but not the direction of the velocity, and this means that the irrotational part of the volume-averaged convective inertial term must be unchanged, but the rotational part is not determined by the averaging process, and there is no inconsistency in setting it to zero as part of the closure process.

In the process of performing the closure after volume-averaging, it has been traditional to adjust for the contribution to the overall drag force, that includes a quadratic drag force that has a specific direction (parallel to the Darcy velocity in the case of an anisotropic medium), but to ignore the fact that one also needs to adjust for the fact that the overall moment of the force system has to be zero. Nield (2000a) suggested that an appropriate adjustment is simply to set to zero the irrotational part of the volume-averaged convective inertial term.

It has sometimes been claimed that the retention of the convective inertial term is necessary in order to account for the formation of hydrodynamic boundary layers in channel flow, and in order to estimate the entrance length, but this is not correct. The formation of such layers is primarily due to the action of viscous diffusion, and the entrance length can be estimated using the time-derivative inertial term.

In many practical cases it does not matter computationally whether the advective inertial term is included or not because, relative to the quadratic drag 
term, it is of order of magnitude $K^{\frac{1}{2}} / c_{F} \phi^{2} L$ (where $L$ is a characteristic length scale), and this is normally small. [Compare Lage (1992).]

This topic is related to the question of how best to model turbulence in a porous medium. This is currently a controversial topic (Nield, 2000b).

3. Brinkman viscous term

Brinkman (1947) introduced the Laplacian viscous term in a restricted context. Its global use is due to other authors. The global treatment may fail to deal adequately with the distinctive features of flow in a porous medium. The ratio of the Brinkman term to the Darcy term is of order $D a=K / L^{2}$ (where $L$ is the appropriate macroscopic length scale), so $D a \rightarrow \infty$ corresponds to a fluid clear of solid material. In most practical cases $D a$ will be very small, and the Brinkman term will have a significant effect only in thin layers (within a dimensional distance of order $K^{\frac{1}{2}}$ of a solid wall. In many cases the reduction in velocity in this thin layer will be masked by an increase in velocity (the channeling effect) due to increase in porosity near the wall (where solid particles cannot pack as tightly as they can in the interior).

The Brinkman equation cannot be rigorously justified except when the porosity is close to unity. The self consistent formulation of Brinkman breaks down when $\phi<0.6$. There is an uncertainty about the effective viscosity $\mu_{e}$. Brinkman took $\mu_{e}=u$. Formal averaging (Bear \& Bachmat, 1990) leads to $\mu_{e}=\mu / \phi T$, where $T$ is the tortuosity . Whitaker (1999, p. 173) ignores the tortuosity. He emphasizes that the Brinkman correction essentially involves the intrinsic velocity, so that when the correction is written in terms of the Darcy velocity this immediately leads to $\mu_{e}=\mu / \phi$. Until recently it had not been possible to check the alternatives against experiment because all the available experimental data pertained to media whose porosity was outside the range for which the theoretical results are valid. Givler \& Altobelli (1994), using NMR, found $\mu_{e}=8 \mu$ approximately for water flowing through a rigid foam material ( $\phi$ $=0.972)$. It is clear that averaging is inadequate in this case.

4. Dupuit-Forchheimer term.

The term $\left(c_{F} \rho / K^{\frac{1}{2}}\right) v \mathbf{v}$ is in the form recommended by Joseph, Nield and Papanicolaou (1982). The scalar form is due to Ward (1964), who thought that $c_{F}$ might be a universal constant, 0.55 . Subsequent experimenters found that $c_{F}$ is approximately constant for a particular family of materials, e.g $c_{F}=0.1$ for foamed metal fibres. A semi-empirical derivation of an estimate for $c_{F}$ was reported by Joseph et al. (1982). They emphasized that the drag is quadratic and in a direction opposed to $\mathbf{v}$ (so one cannot write down uncoupled equations for the $x$ - and $y$-components of $\mathbf{v}$ ). In fact, a cubic drag term arises in two circumstances. First, Lage et al. (1997) pointed out that, when complications resulting from transition to turbulence are taken into account, the coefficient of the quadratic term varies slowly with velocity, and so the drag is effectively cubic within a restricted Reynolds number range. Second, Mei and Auriault 
(1991) and others have pointed out that in the weak inertial regime (one for which the pore Reynolds number is less than unity, the variation from the linear term is in fact cubic, rather than quadratic. Antohe and Lage (1999) have argued that the factor $K^{\frac{1}{2}}$ in the Dupuit-Forchheimer term is better replaced by another quantity having the dimensions of length, namely a typical particle diameter.

\section{Modelling a porous-medium/clear-fluid interface}

Since the differential equations for the two regions are of second order in spatial derivatives, four matching conditions are needed if the Brinkman equation is employed. These involve the continuity of tangential velocity, normal velocity, tangential stress and normal stress. The velocity matching causes no problems, but with the stress matching it is different. Consider the matching of tangential stress. Over the pore portion of the interface the velocity shear, and hence the tangential stress, is continuous. Over the solid portion the tangential shear is not continuous; it is obviously zero in the solid, but has some indeterminant non-zero value in the adjacent clear fluid. Authors who have matched velocity shears have overdetermined the system of equations.

When one uses the Darcy equation (instead of the Brinkman equation) in the porous medium the difficulty can be side-stepped. Now one needs only three matching conditions; two of these are the continuity of tangential velocity and normal velocity, and the Beavers-Joseph (1967) boundary condition:

$$
\frac{u_{f}}{y}=\frac{\alpha_{B J}}{K^{\frac{1}{2}}}\left(u_{f}-u_{m}\right) .
$$

Here the clear fluid occupies the region ( $\mathrm{y}>0$ ), and $u_{f}$ is the fluid velocity, and $u_{f}$ and $\partial u_{f} / \partial y$ are evaluated at $y=0+$. The Darcy velocity $u_{m}$ is evaluated at some small distance from $y=0$. The Beavers-Joseph constant $\alpha_{B J}$ is dimensionless and independent of the fluid viscosity, but it depends on the structure of the porous material within the boundary region. Sahraoui \& Kaviany (1992) have shown that the value of $\alpha_{B J}$ depends on the flow direction at the interface, the Reynolds number, the extent of the clear fluid, and nonuniformities in the arrangement of solid material at the surface. It seems best to regard $\alpha_{B J}$ as an empirical constant, to be determined experimentally. Its presence in the boundary condition provides the needed flexibility in modelling the tangential stress requirement.

The situation with respect to the normal stress is similar, but there is an additional factor involved. The normal stress is the sum of the pressure and a viscous stress term. Some authors have argued that the pressure, being an intrinsic quantity, has to be continuous across the interface. They have failed to realize that the interface is an idealization of a thin layer in which the pressure can change substantially because of the presence of the solid material. In practice, the viscous term in the normal stress may be small compared with the pressure, and in this case the continuity of normal stress does reduce to the approximate continuity of pressure. 
Also, for an incompressible fluid, the continuity of normal stress does reduce to continuity of pressure if one takes the effective Brinkman viscosity equal to the fluid viscosity, as shown by Chen and Chen (1992). Authors who have formulated a problem in terms of stream function and vorticity have failed to deal properly with the normal stress boundary condition (Nield, 1997). For a more soundly based procedure for numerical simulation, and for a further discussion of this matter, the reader is referred to Gartling et al. (1996).

Ochoa-Tapia and Whitaker (1995a,b) have expressly matched the Darcy and Stokes equations using the volume-averaging procedure. This approach produces a jump in the stress (but not in the velocity) and involves a parameter to be fitted experimentally. They also explored the use of a variable porosity model as a substitute for the jump condition, and concluded that the latter approach does not lead to a successful representation of all the experimental data but it provides insight into the complexity of the interface region. Kuznetsov (1996) applied the jump condition to flows in parallel-plate and cylindrical channels partially filled with a porous medium. Kuznetsov (1997) reported an analytical solution for flow near an interface.

Salinger et al. (1994) found that a Darcy-slip finite-element formulation produced solutions which were more accurate and more economical to compute than those obtained using a Brinkman formulation.

\section{Non-Newtonian fluid}

Shenoy (1994) reviewed studies of flow of non-Newtonian fluids in porous media. Attention has been concentrated on power-law fluids. Shenoy suggested, on the basis of volumetric averaging, that the Darcy term be replaced by $\left(\mu^{*} / K^{*}\right) v^{n-1} \mathbf{v}$, the Brinkman term by $\left(\mu^{*} / \phi^{n}\right) \nabla\left\{\left|\sqrt{ }\left[\frac{1}{2} \Delta: \Delta\right]\right|^{n-1} \nabla\right\}$ for an Ostwald-de Waele fluid, and the Forchheimer term be left unchanged (because it is independent of the viscosity). Here $n$ is the power-law index, $\mu^{*}$ reflects the consistency of the fluid, $K^{*}$ is a modified permeability, and $\Delta$ is the deformation tensor. The author agrees with Shenoy's suggestion, but in the Brinkman term he would replace $\left(\mu^{*} / \phi^{n}\right)$ by an equivalent coefficient.

\section{Effect of rotation}

The effect of rotation is to add extra body-force terms to the momentum equation, reflecting the centrifugal and Coriolis effects. In the context of natural convection, the topic has been discussed in papers reviewed by Vadasz $(1997,1998)$. The lefthand side of Equation (3) is replaced by

$$
\rho\left[\frac{\mathbf{v}}{\phi t}+\frac{1}{\phi^{2}}(\mathbf{v} \cdot \nabla) \mathbf{v}+\frac{2}{\phi} \omega \times \mathbf{v}+\omega \times(\omega \times \mathbf{x})\right]
$$


where $\omega$ is the angular velocity of the rotating frame of reference and $\mathbf{x}$ is the position vector relative to that frame. The ratio of the Coriolis term to the Darcy term is of order $E^{-1}$, where the Ekman-Darcy number $E$ is given by

$$
E=\phi E k / D a, \quad E k=\mu / 2 \omega \rho L^{2}, \quad D a=K / L^{2} .
$$

Here $L$ is a characteristic length. In most practical situations $E$ is large, generally because the Darcy number $D a$ is small, so the Coriolis term is usually not important. However, Vadasz points out that in the case of heterogenous media the Coriolis acceleration distorts the direction of any existing flow and generates vortices in a plane perpendicular to the flow. For isothermal fluids the centrifugal term, being irrotational, merely affects a reduced pressure, but for free convection this term may be important.

Many authors have wrongly omitted the factor $\phi$ from the Coriolis term. As Nield (1999) pointed out, they failed to realize that the pressure in Darcy's equation is an intrinsic quantity, and hence all the velocity appearing in an inertial term must also be an intrinsic quantity. In fact, Nield has suggested that it would be simpler and less confusing to rewrite the momentum equation in terms of the intrinsic velocity and with the permeability $K$ replaced by the "retardability" $R$, defined by $R=\phi / K$. This proposed change has other advantages. The new "effective viscosity" becomes close to the fluid viscosity and the new Forchheimer coefficient becomes closer to being a universal constant.

\section{Effect of a magnetic field}

The technique of volume-averaging leads to the prediction that the effect of a magnetic field is to add a body-force term $\sigma(\mathbf{v} \times \mathbf{B}) \times \mathbf{B} / \phi$ to the right-hand side of Equation (2). Here $\sigma$ is the electical conductivity of the fluid and $\mathbf{B}$ is the applied magnetic induction. (See, for example, Raptis \& Perdikis (1987).) In the case of two-dimensional flow and with the magnetic induction in the plane of that flow, the extra body force reduces to $\left(-\sigma B^{2}\right) \mathbf{v} / \phi$. Thus the effect of the magnetic field is then simply to add an additional drag force. The ratio of the magnetic drag to the Darcy drag is $\sigma \mathbf{B}^{2} K / \mu \phi$. In most practical cases this Chandrasekhar-Darcy number is very small, so the effect to the magnetic field is negligible. Again, many authors have omitted the factor $\phi$ in error.

\section{Viscous dissipation}

For convection problems one must supplement the momentum equation by a thermal energy equation, which in steady state form is

$$
\rho c_{p} \mathbf{v} \cdot \nabla T=\nabla \cdot\left(k_{e} \nabla T\right)+\Phi
$$

where $k_{e}$ is the effective thermal conductivity of the porous medium and $\Phi$ is the viscous dissipation term. This last term is generally negligible, but in general is 
given by the power of the drag force (per unit volume), i. e. $\mathbf{v} \cdot \mathbf{F}$ where $\mathbf{F}$ is the drag force. In the case where Eq. (3) applies we have

$$
\Phi=\frac{\mu}{K} \mathbf{v} \cdot \mathbf{v}+\frac{c_{F} \rho}{K^{\frac{1}{2}}}|\mathbf{v}| \mathbf{v} \cdot \mathbf{v} .
$$

The remarkable thing is that the last term does not involve the viscosity as a factor, despite the fact that it contributes to the viscous dissipation term. This paradox was resolved by Nield (2000a). The short explanation is that the inertial effects are mediated by the pressure distribution and this affects the velocity field and hence the drag in a complex fashion. Boundary layer separation and wake formation are involved in the explanation.

\section{References}

1. B.V. Antohe. and J. L. Lage, Darcy's experiments and the transition to nonlinear flow regime. Proceedings of the 33rd National Heat Transfer Conference, August 15-17, 1999, Albuquerque, New Mexico, NHTC99-180, pp. 1-7, 1999.

2. J. Bear and Y. Bachmat, Introduction to Modeling of Transport Phenomena in Porous Media. Kluwer Academic, Dordrecht, 1990.

3. G. S. Beavers and D. D. Joseph, Boundary conditions at a naturally permeable wall. J. Fluid Mech. 30: 197-207, 1967.

4. H. C. Brinkman, A calculation of the viscous force exerted by a flowing fluid on a dense swarm of particles. Appl. Sci. Res. A, 1: 27-34, 1947.

5. F. Chen and C. F. Chen, Convection in superposed fluid and porous layers. J. Fluid Mech. 234: 97-119, 1992.

6. H. P. C. Darcy, Les Fontsines Publiques de la Ville de Dijon. Victor Dalmont, Paris, 1856.

7. J. Dupuit, Etudes Theoriques et Pratiques sur le Mouvement des Eaux. Dunod, Paris, 1863.

8. P. Forchheimer, Wasserbewegung durch Boden. VDI Z., 45: 1782-1788, 1901.

9. D. K. Gartling, C. E. Hickox and R. C. Givler, Simulation of coupled viscous and porous flow problems. Comp. Fluid Dyn. 7: 23-48, 1996.

10. R. C. Givler and S. A. Altobelli, A determination of the effective viscosity for the BrinkmanForchheimer flow model. J. Fluid Mech. 258; 355-370, 1994.

11. C. T. Hsu and P. Cheng, Thermal dispersion in a porous medium. Int. J. Heat Mass Transfer, 33: 1587-1597, 1990.

12. D. D. Joseph, D. A. Nield and G. Papanicolaou, Nonlinear equation governing flow in a saturated porous medium. Water Resources Research, 18: 1049-1052 and 19: 591, 1982.

13. M. Kaviany, Principles of Heat Transfer in Porous Media. Springer, New York, 1991.

14. A. V. Kuznetsov, Analytical investigation of the fluid flow in the interface region between a porous medium and a clear fluid in channels partially filled with a porous medium. Appl. Sci. Res.. 56: 53-57 1997.

15. A. V. Kuznetsov, Influence of the stress jump condition at the porous-medium/clear-fluid interface on a flow at a porous wall. Int. Comm. Heat Mass Transfer 24: 401-410, 1997.

16. J. L. Lage, Effect of the convective inertia term on Bénard convection in a porous medium. Num. Heat Transfer A, 22: 469-485, 1992.

17. J. L. Lage, The fundamental theory of flow through permeable media from Darcy to turbulence. Transport Phenomena in Porous Media (D. B. Ingham \& I. Pop, Eds.) Elsevier Science, Oxford, pp. 1-30, 1998.

18. J. L. Lage, B. V. Antohe and D. A. Nield, Two types of nonlinear pressure-drop vesrus flowrate relation observed for saturated porous media. ASME J. Fluids Engng. 119: 701-706, 1997.

19. C. C. Mei and J. L. Auriault, The effect of weak inertia on flow through a porous medium. J. Fluid Mech. 222: 647-663, 1991. 
20. D. A. Nield, The limitations of the Brinkman-Forchheimer equation in modelling flow in a saturated porous medium and at an interface. Int. J. Heat Fluid Flow 12: 269-272, 1991.

21. D. A. Nield, Modelling high speed flow of an incompressible fluid in a saturated porous medium. Transport in Porous Media 14: 85-88, 1994.

22. D. A. Nield, Discussion of a Discussion by F. Chen and C. F. Chen. ASME J. Heat Transfer 119: 193-194, 1997.

23. D. A. Nield, Modeling the effects of a magnetic field or rotation on flow in a porous medium: momentum equation and anisotropic permeability analogy. Int. J. Heat Mass Transfer 42: 3715-3718, 1999.

24. D. A. Nield, Resolution of a paradox involving viscous dissipation and nonlinear drag in a porous medium. Transport in Porous Media. (in press), 2000a.

25. D. A. Nield, Alternative models of turbulence in a porous medium, and related matters. Submitted to ASME J. Fluids Engng. 2000b.

26. D.A. Nield and A. Bejan, Convection in Porous Media. 2nd Ed., Springer, New York, 1999.

27. J. A. Ochoa-Tapia and S. Whitaker, Momentum transfer at the boundary between a porous medium and a homogeneous fluid? I. Theoretical development. Int. J. Heat Mass Transfer 38: 2635-2646, 1995a.

28. J. A. Ochoa-Tapia and S. Whitaker, Momentum transfer at the boundary between a porous medium and a homogeneous fluid? II. Comparison with experiment. Int. J. Heat Mass Transfer 38: 2647-2655, 1995b.

29. A. Raptis and C. Perdikis, Hydromagnetic free-convective flow through porous media. Encyclopedia of Fluid Mechanics and Modeling. (N.P. Cheremisinoff, Editor), Gulf Publishing Co., Houston, Chapter 8, pp. 239-262, 1987.

30. M. Sahraoui and M. Kaviany, Slip and no-slip velocity boundary conditions at the interface of porous, plain media. Int. J. Heat Mass Transfer, 35: 927-943, 1992.

31. A. G. Salinger, R. Aris and J. J. Derby, Finite element formulations for large-scale coupled flows in adjacent porous and open fluid domains. Int. J. Numer. Meth. Fluids 18: 1185-1209, 1994.

32. A. V. Shenoy, Non-Newtonian fluid heat transfer in porous media. Advances in Heat Transfer, 24: 101-190, 1994.

33. P. Vadasz, Flow in rotating porous media. Fluid Transport in Porous Media (ed. P. du Plessis). Computational Mechanics publications, Southampton, Chapter 4, 1999.

34. P. Vadasz, Free convection in rotaing porous media. Transport Phenomena in Porous media (eds. D. B. Ingham and I. Pop), Elsevier, Oxford, 1998.

35. K. Vafai and S. J. Kim, Fluid mechanics of an interface region between a porous medium and a fluid layer-an exact solution. Int. J. Heat Fluid Flow 11: 254-256, 1990.

36. J. C. Ward, Turbulent flow in porous media. J. Hydaul. Div. Am. Soc. Civ. Eng. 90 (HY5): 1-12, 1964.

37. S. Whitaker, The Method of Volume Averaging. Kluwer, Dordrect, 1999. 


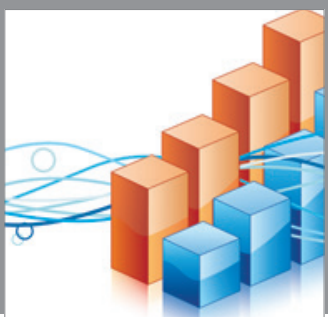

Advances in

Operations Research

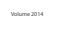

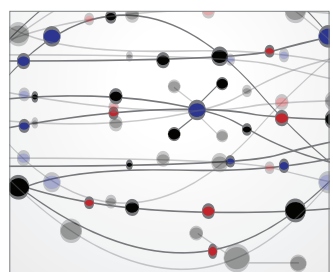

\section{The Scientific} World Journal
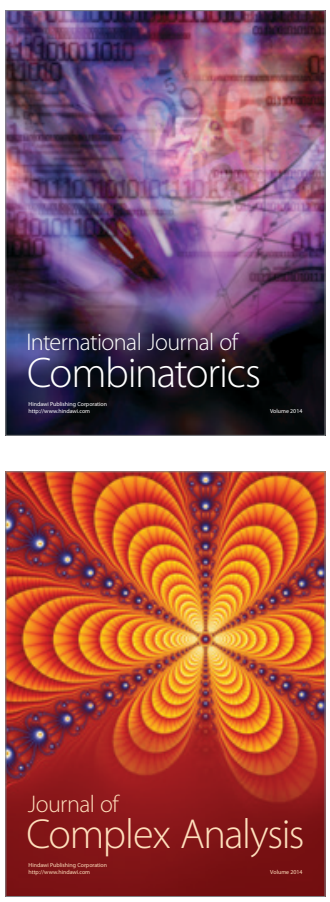

International Journal of

Mathematics and

Mathematical

Sciences
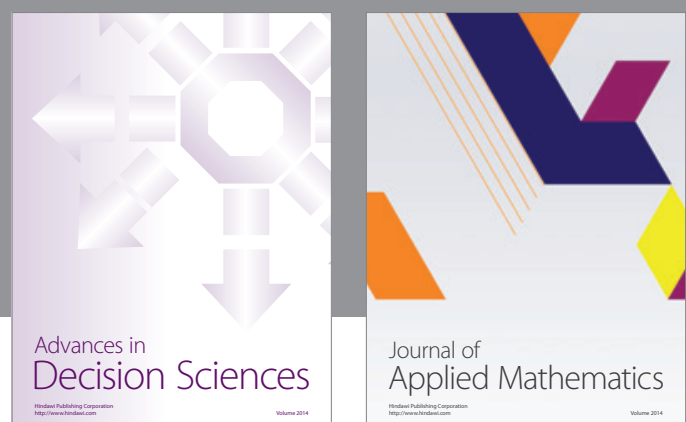

Journal of

Applied Mathematics
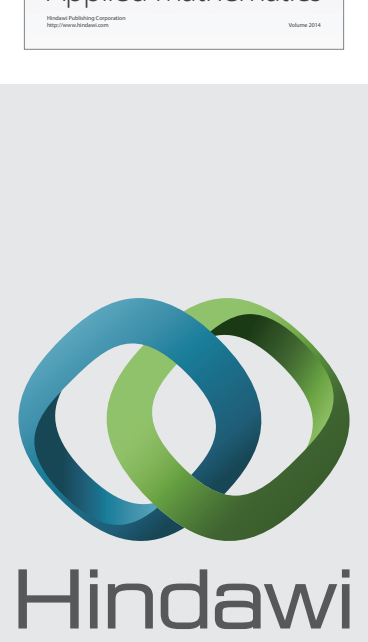

Submit your manuscripts at http://www.hindawi.com
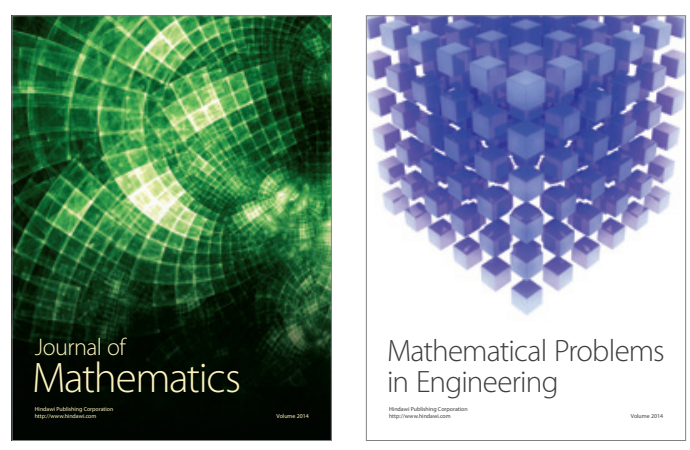

Mathematical Problems in Engineering
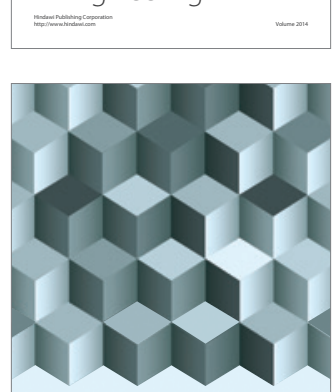

Journal of

Function Spaces
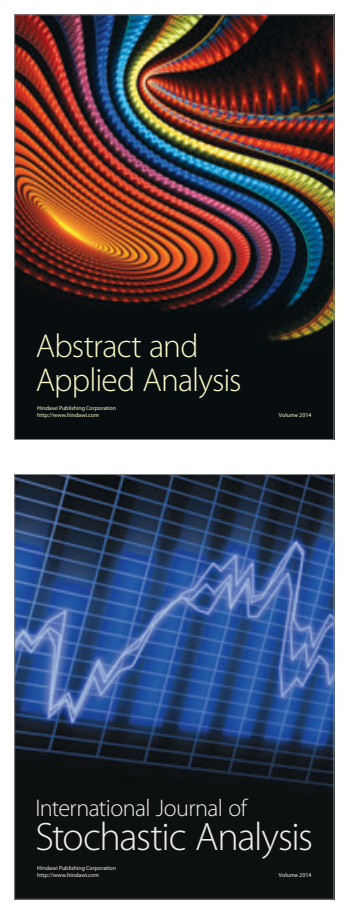

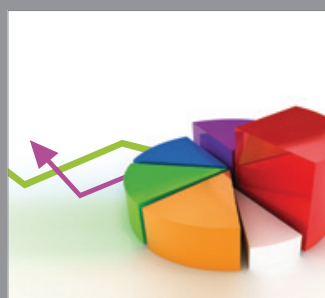

ournal of

Probability and Statistics

Promensencen
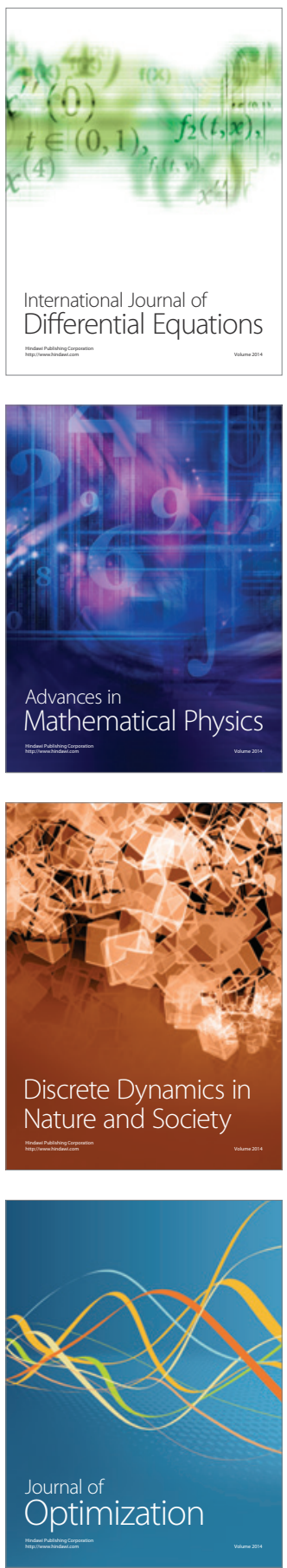